\title{
Aprendiendo el oficio docente. El modelo Lesson Study para mejorar la docencia en la universidad
}

\section{Learning to teach. The Lesson Study model to improve university teaching}

\author{
Adelina Calvo Salvador ${ }^{1}$ \\ calvoa@unican.es \\ IgnaCiO Haya Salmón \\ hayai@unican.es \\ Carlos Rodríguez-Hoyos \\ rodriguezhc@unican.es \\ Universidad de Cantabria, España
}

\section{Resumen:}

Se presentan los resultados parciales de un proyecto de investigación desarrollado durante dos cursos académicos (2015-16 y 2016-17) en las Facultades de Educación y Formación del Profesorado de las Universidades de Cantabria y Oviedo. El objetivo era mejorar la docencia en la universidad en varias asignaturas de diferentes planes de estudio del campo de la educación, a partir del análisis de las prácticas de aula. Para ello, se adoptó una metodología de investigación inspirada en el modelo Lesson Study. Los resultados muestran que los estudiantes aprenden ejercitando competencias que hoy se consideran clave para los docentes. Las conclusiones constatan las potencialidades de este modelo para la mejora de la docencia en la educación superior, subrayando la necesidad de que los estudiantes tengan un papel más activo en todo el proceso.

\begin{abstract}
:
This paper presents the partial results of a research project developed over two academic years (2015-16 and 2016-17) in the Faculties of Education and Teacher Training at the Universities of Cantabria and Oviedo. The objective of the project was to improve teaching in several subjects from different curricula in the field of education, based on the analysis of classroom activities. To this end, a methodology inspired by the Lesson Study model was adopted. The results show that students learn through the use of skills which are considered key for teachers. The conclusions demonstrate that the research process has great potential for improving teaching in higher education and highlight the need for students to assume a more active role throughout the whole research process.
\end{abstract}

1 Dirección para correspondencia (correspondence address):

Adelina Calvo Salvador. Universidad de Cantabria. Facultad de Educación. Edificio Interfacultativo. Avda. de los Castros, s/n. 39005, Santander (España). 
Aprendiendo el oficio docente. El modelo Lesson Study para mejorar la docencia en la universidad

Adelina Calvo Salvador, Ignacio Haya Salmón y Carlos Rodríguez-Hoyos

\section{Palabras clave:}

Lesson Study; educación superior; innovaciones educativas; desarrollo profesional.

\section{Key words:}

Lesson Study; higher education; educational innovations; career development.

\section{Resumée:}

Cet article présente les résultats partiels d'un projet de recherche développé pendant deux années scolaires (2015-16 et 2016-17) dans les Facultés de Pédagogique et Formation des Enseignants dans les universités de Cantabria et Oviedo. L'objectif était d'améliorer I'enseignement à l'université avec l'analyse des pratiques de classe. A été adoptée une méthodologique d'enquête inspirée du modèle LessonStudy. Les résultats montrent que les étudiants apprennent en exerçant des compétences considérées comme très importantes pour les enseignants. Les conclusions soulignent les potentialités du modèle d'améliorer l'enseignement à l'université et la nécessité qu'ont les étudiants d'avoir un rôle plus actif tout au long du processus.

\section{Mots Clés:}

Lesson Study; enseignement supérieur; innovations pédagogiques; développement professionnel.

Fecha de recepción: 06-09-2018

Fecha de aceptación: 13-06-2019

\section{Introducción}

Existe un modelo definido a nivel teórico sobre cuáles son las competencias y saberes que se consideran más relevantes en un "docente competente" como la competencia comunicativa, interpersonal, metodológica, de planificación y gestión de la docencia, de innovación y de trabajo en equipo. Sin embargo, el análisis de las prácticas educativas reales muestra que en la actualidad, los profesionales que son considerados los mejores docentes por su propia universidad, siguen percibiendo la enseñanza más como una tarea solitaria que como una tarea en equipo y focalizan su trabajo en ayudar al alumnado a aprender, más que a desplegar estrategias que le permitan ser co-partícipe de su aprendizaje (Monereo y Domínguez, 2014).

El desarrollo profesional docente en la educación superior no se adquiere sólo con el hecho de aumentar la formación recibida, sino que se requiere modificar su orientación. Esta formación debe trascender las actividades de actualización de competencias docentes para promover un aprendizaje "desde" y "en" la práctica, lo que sin duda aumentará la agencia profesional o capacidad de acción docente. El impacto de esta formación será mayor si se articula en red, en forma de comunidades de 
aprendizaje o comunidades de práctica y si se orienta hacia la mejora de los aprendizajes del alumnado (Ruè, 2015). La formación debe tener en cuenta la cultura profesional de la propia universidad, vincularse a la práctica y favorecer que los docentes puedan enfrentar los dilemas de su trabajo diario (Mas Torelló y Olmos-Rueda, 2016).

La innovación educativa en la enseñanza superior requiere fortalecer los vínculos entre la docencia y la investigación (Cunha, 2015, Pétriz y Rubiralta, 2017), mejorar la coordinación docente, tanto a nivel estructural como en los procesos de enseñanza-aprendizaje (Bolarín, 2016), así como reconsiderar el papel central de los estudiantes en los procesos educativos y el rol que juegan las prácticas de aula (Jiménez, 2017). En un estudio realizado entre egresados de ciclo corto y largo en toda Europa dirigido a valorar la satisfacción que los jóvenes manifestaban en relación a su proceso de aprendizaje y a la formación universitaria recibida, se concluye que las diferencias más Ilamativas de nuestro sistema universitario en relación a otros sistemas europeos se focalizan en la metodología de enseñanza utilizada. En nuestro país se percibe que el docente sigue siendo la fuente de información principal. También se considera que la enseñanza sigue siendo eminentemente teórica, con un peso todavía menor de la enseñanza más práctica (Estudio Reflex, 2007, citado en Martínez, 2017; Montero, 2018).

En la línea de investigaciones anteriores, este proyecto persigue mejorar la docencia en la universidad a partir de la investigación de la enseñanza (Braga, Verdeja y Calvo, 2018; Caballero y Bolívar, 2015; Calvo y Rodríguez-Hoyos, 2017; Martínez Rodríguez, 2011; Pérez Gómez y Soto, 2011). Se pretende con ello promocionar una capacitación docente orientada por lo que ocurre en el contexto real de los procesos educativos teniendo en cuenta la visión que los propios estudiantes ofrecen sobre la educación que reciben, así como la creación de pequeños equipos de trabajo e investigación que acompañen a cada docente en su mejora.

En este marco general, se presentan aquí los resultados parciales de una investigación más amplia que ha sido financiada por la Universidad de Cantabria en su III Convocatoria de Innovación Docente. La metodología de investigación es cualitativa y se enmarca dentro del modelo Lesson Study que ha sido reconocido como una modalidad de la Investigación-Acción. Docentes y estudiantes han tenido un importante papel como agentes de mejora educativa a través de una sistemática recogida 
Aprendiendo el oficio docente. El modelo Lesson Study para mejorar la docencia en la universidad

Adelina Calvo Salvador, Ignacio Haya Salmón y Carlos Rodríguez-Hoyos

de datos, análisis y propuestas de mejora. El trabajo se ha desarrollado durante dos cursos académicos (2015-16 y 2016-17) en las Facultades de Educación y Formación del Profesorado de las dos universidades implicadas.

\section{Un proyecto inspirado en el modelo Lesson Study (LS)}

El modelo Lesson Study, conocido en español como Lecciones de Estudio o Lecciones de Investigación tiene su origen en el marco de las políticas de extensión de la educación primaria y de perfeccionamiento docente en Japón a principios del siglo XX (Soto y Pérez Gómez, 2015). A pesar de sus potencialidades, el modelo tardaría casi un siglo en llegar a Europa, pero a partir de ese momento conocería una rápida expansión por países de diferentes continentes, lo que ha generado, por un lado, una gran diversidad de prácticas que responden a diferentes modelos curriculares y por otro, la constatación de que el modelo es válido para producir cambios en los procesos educativos encaminados a mejorar los resultados de aprendizaje.

Por ejemplo, en el Reino Unido las LS se han establecido a nivel nacional (a través de la National Primary Strategy) de manera que en estos momentos el modelo está bastante generalizado como estrategia de desarrollo docente (Dudley, 2012 2011), aunque el apoyo del gobierno a este modelo ha decaído. Peter Dudley, el director de la citada estrategia, explica que su diseño fue posible a partir de diferentes cambios que se fueron dando en la política educativa del Reino Unido en la educación primaria y que supusieron, progresivamente, el establecimiento de una relación entre los buenos resultados de los estudiantes y la formación de los docentes y la evidencia de que, para seguir mejorando esos resultados y hacerlos sostenibles en el tiempo, era necesario proponer recursos y herramientas que lograsen capacitar a las escuelas y sus docentes en la enseñanza y en el liderazgo. Es así como fue posible un primer estudio piloto a nivel nacional entre los años 2003 y 2005 donde, a través de diferentes sistemas de consulta al alumnado, se obtuvieron valiosas ideas sobre cómo mejorar la enseñanza y el aprendizaje. La estrategia de unas lecciones planificadas, observadas y analizadas conjuntamente estaban en la base de ese primer proyecto piloto que posteriormente se difundiría a nivel nacional y que tomaría forma en la ya citada National 
Primary Strategy. Aunque el autor es muy crítico con la falta de apoyo gubernamental a la Strategy a partir del 2015, no es menos cierto que el modelo LS no ha dejado de crecer, implicando en la actualidad a cada vez más escuelas y universidades (Dudley, 2015).

Una situación similar se da en Suecia, donde las LS se han introducido desde el 2003 a través de un proyecto de investigación fundado por el Swedish Research Council. Desde entonces, se estima que cientos de escuelas han implantado, en diferente grado, el modelo LS. Precisamente en este país se ha implementado un tipo de LS que utiliza la teoría de la variación (teoría de aprendizaje) como principio que orienta el diseño y el análisis de los procesos de aprendizaje en las aulas (Holmqvist, 2015).

Al mismo tiempo, a nivel europeo se está creando una red de trabajo que se está viendo favorecida por el desarrollo de plataformas online y sistemas específicos de grabación de video que permiten al profesorado compartir e intercambiar sus prácticas. La página web promovida por el académico Peter Dudley (http://lessonstudy.co.uk) donde se accede a un manual en varios idiomas sobre cómo implantar las LS en escuelas, tiene un número de visitantes que no deja de crecer. Al mismo tiempo, se ha señalado la importancia de cómo inventar un camino para incorporar la práctica reflexiva en las rutinas y hábitos de la vida profesional de las escuelas europeas, algo que ya se está haciendo realidad, gracias al papel activo de escuelas y docentes que trabajan diariamente por identificar a otros centros y profesionales que estén en disposición de experimentar las LS. De manera más concreta se ha establecido una hoja de ruta con cinco grandes pasos para ir consolidando esta red a nivel europeo: (1) Crear en cada país o comunidad sistemas pilotos; (2) Desarrollar un plan para generalizar los resultados a partir de las experiencias piloto; (3) Involucrar a los líderes (personas más experimentadas en las LS y personas en cargos de gestión educativa) en las LS; (4) Buscar conocimiento especializado y probado de alta calidad y (5) Difundir las prácticas exitosas (Dudley, 2015).

La red de trabajo de escuelas, universidades y profesionales vinculados a las LS tiene un alcance mundial, como demuestra la existencia de la World Association for Lesson Studies (WALS) con sede en Singapur y la actividad que la misma despliega a través de publicaciones y congresos científicos. El modelo también ha sido utilizado en la educación superior, tanto en estudios de grado como de máster y en ámbitos como la formación inicial, la formación permanente del profesorado y la investigación 
Aprendiendo el oficio docente. El modelo Lesson Study para mejorar la docencia en la universidad

Adelina Calvo Salvador, Ignacio Haya Salmón y Carlos Rodríguez-Hoyos

educativa (Dudley, 2015; Peña y Pérez Gómez, 2019; Soto, Serván y Pérez Gómez, 2015; Soto, Serván y Caparrós, 2016; Wood and Cajkler, 2016).

El modelo LS permite cambiar la educación promoviendo una mejora educativa que viene desde los docentes, que se articula de "abajo-arriba" y que promueve una vía efectiva para la mejora de la enseñanza y el aprendizaje a través del intercambio de conocimiento profesional sobre la enseñanza. Promueve un desarrollo profesional dirigido por interrogantes (no por respuestas cerradas), protagonizado por los participantes (no por expertos externos), a través de una comunicación entre docentes (no desde el experto o formador externo hacia el docente), promoviendo relaciones recíprocas entre docentes (no jerárquicas) e investigando sus propias prácticas (no desde la creencia de que la investigación informa las prácticas) (Lewis, 2002).

En nuestro caso, el modelo LS se vincula a la Investigación-Acción y a la idea del docente como investigador (Elliot, 2015). Se diseñó un proyecto de investigación dirigido a contestar esta pregunta: “¿Cómo puedo hacer que mis estudiantes aprendan mejor en la universidad a través de las prácticas de aula?". El proyecto fue desarrollado por docentes e investigadores de las Universidades de Cantabria y de Oviedo (de las Facultades de Educación y Formación del Profesorado) a través de diferentes asignaturas de distintas titulaciones del campo de la educación y durante dos cursos académicos. En cada asignatura, considerada como un caso de estudio, se aplicó el modelo de las LS con el objetivo general de valorar el impacto, recepción y utilidad para el aprendizaje de los estudiantes de las prácticas evaluables de cada asignatura. En cada caso se organizaron equipos de trabajo en los que los estudiantes se incorporan a la investigación como "amigos críticos" (Escudero, 2009). En este artículo se presentan los resultados del trabajo desarrollado en la Universidad de Cantabria durante el curso 2015-16. La metodología de investigación tiene una clara inspiración cualitativa (Flick, 2014).

\section{Objetivos de la investigación}

La finalidad general de este proyecto era lograr un conocimiento profundo y complejo sobre cómo se estaban desarrollando las prácticas de aula con el objetivo de mejorar los aprendizajes de los estudiantes. Esta finalidad general se concretó en los siguientes objetivos específicos: 
- Conocer la utilidad de las prácticas de aula para promover competencias docentes que permitan una formación inicial en un contexto de incertidumbre, complejidad y donde se perciba la necesidad de vincularse al cambio y la mejora.

- Examinar el valor del diseño y desarrollo de las prácticas de aula (tomadas aquí como lección de estudio) para promover el desarrollo profesional del profesorado universitario y una mejora de su docencia en la universidad.

- Analizar y poner en marcha las mejoras que se derivan de todo el proceso y valorar en qué medida impactan en el currículum universitario y en la cultura de la institución.

\section{Metodología}

Tal y como se ha constatado en trabajos anteriores, el modelo LS propone una metodología para investigar la práctica educativa que proviene de la Investigación-Acción, lo que supone que las fases de trabajo se organizan a partir de un ciclo de reflexión y acción (Hurd y LicciardoMusso, 2005; Pérez Gómez y Soto, 2011; Soto y Pérez Gómez, 2015). En cada caso-asignatura, las prácticas de aula han sido tomadas como una lección de estudio, para lo que se ha formado un equipo de tres docentes que impulsa el trabajo conjunto y la reflexión. Cada caso es entendido aquí como un sistema único, específico y complejo que debe ser analizado para conocer qué le hace único y qué tiene en común con el resto de casos analizados (Stake, 1998).

\subsection{Presentación de cada caso-asignatura}

En cada caso-asignatura, la LS se aplicó a una práctica evaluable de clase que se consideraba central, por su complejidad, duración, por la necesidad de movilizar diferentes conocimientos teóricos-prácticas para su resolución y por su peso en la calificación final del alumnado: al menos el $40 \%$ de su nota final. La LS se desplegó en cada una de las tres asignaturas implicadas durante el primer año del proyecto y en la Universidad de Cantabria. A continuación, se muestra una tabla-resumen de cada uno de los casos en los que se aplicó la LS, todos ellos en el marco del Grado de Educación Infantil. 
Aprendiendo el oficio docente. El modelo Lesson Study para mejorar la docencia en la universidad

Adelina Calvo Salvador, Ignacio Haya Salmón y Carlos Rodríguez-Hoyos

Tabla 1. Elaboración propia

Casos de investigación

\begin{tabular}{|c|c|c|c|}
\hline Asignatura & Curso & Breve descripción de la práctica & $\begin{array}{c}\text { Sesiones de la } \\
\text { práctica }\end{array}$ \\
\hline $\begin{array}{l}\text { El centro } \\
\text { escolar como } \\
\text { comunidad } \\
\text { educativa }\end{array}$ & $2^{\circ}$ curso & $\begin{array}{l}\text { Elaboración de un plan de mejora } \\
\text { de centro a partir de un diagnósti- } \\
\text { co vivencial basado en las prácti- } \\
\text { cas del Grado, en las experiencias } \\
\text { vividas como estudiantes y en las } \\
\text { preocupaciones pedagógicas ac- } \\
\text { tuales como docente en formación }\end{array}$ & $\begin{array}{l}4 \text { sesiones de } 2 \\
\text { horas cada una }\end{array}$ \\
\hline $\begin{array}{l}\text { Tecnología } \\
\text { Educativa }\end{array}$ & $3^{\circ}$ curso & $\begin{array}{l}\text { Elaboración de una narrativa } \\
\text { transmedia: uso de imagen fija } \\
\text { (tres fotos) y en movimiento (crea- } \\
\text { ción de un corto) para elaborar } \\
\text { una campaña de publicidad que } \\
\text { denuncie un tema social relevante } \\
\text { (p.e. desigualdad de género, co- } \\
\text { mercio justo, etc.) }\end{array}$ & $\begin{array}{l}8 \text { sesiones de } 2 \\
\text { horas cada una }\end{array}$ \\
\hline $\begin{array}{l}\text { Fundamentos } \\
\text { Pedagógicos } \\
\text { de Atención a } \\
\text { la Diversidad }\end{array}$ & $3^{\circ}$ curso & $\begin{array}{l}\text { Análisis grupal de } 4 \text { experiencias } \\
\text { educativas reales, documentadas } \\
\text { en revistas de divulgación pedagó- } \\
\text { gica y que responden a la filosofía } \\
\text { del concepto "escuelas y aulas en } \\
\text { movimiento" }\end{array}$ & $\begin{array}{l}6 \text { sesiones de } 2 \\
\text { horas cada una }\end{array}$ \\
\hline
\end{tabular}

\subsection{Fases y técnicas de recogida de datos}

En nuestro proyecto, las fases de investigación han sido un total de cinco: (1) Constitución del grupo de investigación y definición del problema a investigar; (2) Diseño conjunto del plan de investigación y discusión de la LS; (3) Desarrollo de la LS y recogida de datos; (4) Análisis de datos, contraste, reflexión, elaboración de un informe final del caso y (5) Nueva definición del problema.

Las técnicas de recogida de datos han sido cuatro. La observación abierta o naturalista de las prácticas de aula (Angrosino, 2012), la redacción de un diario de campo (Zabalza, 2004), el grupo de discusión (GD) (Barbour, 2013) y la entrevista final al docente (Kvale, 2011).

Cabe señalar que el total de observaciones en cada caso-asignatura ha sido de tres correspondientes a los tres momentos clave durante el desarrollo de las prácticas (inicial, medio y final). Cada sesión de obser- 
vación estuvo documentada a través de la recogida de notas de campo y la redacción de un diario y fue desarrollada por uno de los dos docentes que conformaban el equipo de investigación y que no era el profesor responsable de la asignatura. Se decidieron desarrollar las tres sesiones de observación señaladas por corresponder las mismas a tres momentos importantes del desarrollo de las prácticas y por alcanzar, con ellas, el nivel de saturación de información con esta técnica (Alonso, 1998).

Una vez finalizada la lección de estudio (las prácticas de aula), se organizó un GD con estudiantes (considerados aquí como amigos críticos) donde también asistió el docente responsable de la asignatura como un participante más. El grupo estuvo conducido por uno de los docentes que observaron las sesiones, y el total de estudiantes que participó en cada grupo de discusión fue de cuatro. Las sesiones fueron grabadas en audio, transcritas y devueltas a cada miembro del grupo por si consideraban pertinente realizar alguna matización final antes de archivar estos datos de forma definitiva para su análisis posterior. Para la realización del GD se partía de un guion previo que se adaptó a las particularidades de cada caso, a la realidad y dilemas que mostraban las observaciones, los diarios de campo y las grabaciones iniciales de las fases I y II de la investigación (ver tabla número 2). El guion inicial del GD comprendía preguntas: (1) Dirigidas a que los estudiantes describieran las prácticas de aula y su sentido y significado en el marco de la asignatura; (2) Orientadas a conocer la valoración que realizan los estudiantes y el docente sobre aspectos organizativos y procedimentales de las prácticas (clima de aula, organización de los grupos, temporalización, etc.); (3) Dirigidas a conocer cuál es el impacto formativo de las prácticas, qué aprendizajes reconocen como valiosos en el marco de la asignatura y en su formación como futuros docentes y (4) Valoración sobre el proceso de la LS.

La recogida de datos finalizó con una entrevista semi-estructurada al docente responsable de la asignatura, también dirigida por uno de los docentes facilitadores (observador) y miembro de su equipo de investigación. Al igual que en la técnica anterior, el guion previo de entrevista sufrió adaptaciones en cada caso, fruto de la realidad y dilemas que mostraban las observaciones, los diarios de campo y las grabaciones iniciales de las fases I y II de la investigación (ver tabla número 2). El guion inicial de la entrevista semi-estructurada comprendía preguntas: (1) Dirigidas a conocer la valoración del docente responsable de la asignatura sobre el GD con el alumnado como estrategia para repensar su 
Aprendiendo el oficio docente. El modelo Lesson Study para mejorar la docencia en la universidad

Adelina Calvo Salvador, Ignacio Haya Salmón y Carlos Rodríguez-Hoyos

práctica docente; (2) Orientadas a recoger las reflexiones, valoración y discusión sobre las opiniones y propuestas de mejora compartidas por los estudiantes; (3) Dirigidas a concretar y sintetizar qué mejoras y cambios concretos pueden ser incorporados en el diseño inicial de la LS y (4) Valoración sobre el proceso de la LS.

En la parte final del ciclo de investigación, cada docente redactó un informe final del caso bajo el siguiente esquema general: (1) Breve descripción de la actividad; (2) Dilemas docentes iniciales y (3) Desarrollo y posible resolución de los dilemas docentes y propuestas para un nuevo ciclo de reflexión.

Un resumen de las fases de investigación y de las técnicas de recogida de datos se refleja en la tabla siguiente:

Tabla 2. Elaboración propia

Resumen de las fases de investigación y recogida de datos

\begin{tabular}{|c|c|c|c|}
\hline Fase & Descripción & Recogida de datos & Implicados \\
\hline 1 & $\begin{array}{l}\text { - Constitución del equi- } \\
\text { po de investigación para } \\
\text { cada caso-asignatura. } \\
\text { - Definición del problema } \\
\text { a investigar: principales } \\
\text { interrogantes sobre las } \\
\text { prácticas de aula. }\end{array}$ & $\begin{array}{l}\text { - Grabación en au- } \\
\text { dio de las reunio- } \\
\text { nes. }\end{array}$ & $\begin{array}{l}\text { - } 3 \text { docentes: pro- } \\
\text { fesor responsable } \\
\text { de la asignatura } \\
\text { (R) y dos observa- } \\
\text { dores }(\mathrm{O})\end{array}$ \\
\hline II & $\begin{array}{l}\text { - Presentación de las prác- } \\
\text { ticas y discusión en el } \\
\text { seno del equipo de inves- } \\
\text { tigación ( } 3 \text { docentes uni- } \\
\text { versitarios). } \\
\text { - Planificación conjunta } \\
\text { de la lección de estudio. } \\
\text { - Planificación de la reco- } \\
\text { gida de datos. }\end{array}$ & $\begin{array}{l}\text { - Grabación en au- } \\
\text { dio de las reunio- } \\
\text { nes. } \\
\text { - Análisis y discu- } \\
\text { sión de la pro- } \\
\text { puesta de práctica } \\
\text { de aula aportada } \\
\text { por el profesor } \\
\text { responsable (R). }\end{array}$ & $\begin{array}{l}\text { - } 3 \text { docentes: pro- } \\
\text { fesor responsable } \\
\text { de la asignatura } \\
\text { (R) y dos observa- } \\
\text { dores (O1 y O2). }\end{array}$ \\
\hline III & $\begin{array}{l}\text { - Desarrollo de la lección } \\
\text { de estudio (prácticas de } \\
\text { aula). } \\
\text { - Recogida de datos a tra- } \\
\text { vés de diferentes técnicas. } \\
\text { - Análisis parcial de los da- } \\
\text { tos y reconsideración del } \\
\text { plan inicial de la recogi- } \\
\text { da de datos. }\end{array}$ & $\begin{array}{l}\text { - Observaciones de } \\
\text { aula (3 en cada } \\
\text { caso-asignatura). } \\
\text { - Redacción del } \\
\text { diario de campo } \\
\text { (3 en cada caso). } \\
\text { - Grupo de discu- } \\
\text { sión (docentes y } \\
\text { estudiantes, } 1 \text { en } \\
\text { cada caso). }\end{array}$ & $\begin{array}{l}\text { - R desarrolla la lec- } \\
\text { ción de estudio y } \\
\text { O1 y O2 realizan } \\
\text { las observaciones } \\
\text { de aula y redactan } \\
\text { el diario. } \\
\text { - Grupo dirigido } \\
\text { por O1 u O2. Par- } \\
\text { ticipan } 4 \text { estudian- } \\
\text { tes y R. }\end{array}$ \\
\hline
\end{tabular}




\begin{tabular}{|c|c|c|c|}
\hline Fase & Descripción & Recogida de datos & Implicados \\
\hline & & $\begin{array}{l}\text { - Entrevista al do- } \\
\text { cente ( } 1 \text { en cada } \\
\text { caso). }\end{array}$ & $\begin{array}{l}\text { - Entrevista realiza- } \\
\text { da por O1 u O2. }\end{array}$ \\
\hline IV & $\begin{array}{l}\text { - Reflexión y análisis de los } \\
\text { datos teniendo en cuenta } \\
\text { los dilemas planteados } \\
\text { en la fase I. }\end{array}$ & $\begin{array}{l}\text { Redacción de un } \\
\text { informe final por } \\
\text { cada caso-asigna- } \\
\text { tura. }\end{array}$ & $\begin{array}{l}\text { - } 3 \text { docentes: pro- } \\
\text { fesor responsable } \\
\text { de la asignatura } \\
(\mathrm{R}) \text { y dos observa- } \\
\text { dores }(\mathrm{O} 1 \text { y } \mathrm{O} 2) \text {. }\end{array}$ \\
\hline V & $\begin{array}{l}\text { - Comienzo un nuevo ci- } \\
\text { clo de investigación. } \\
\text { - Nueva definición del } \\
\text { problema. }\end{array}$ & $\begin{array}{l}\text { Informe final por } \\
\text { cada caso-asigna- } \\
\text { tura. }\end{array}$ & $\begin{array}{l}\text { - } 3 \text { docentes: pro- } \\
\text { fesor responsable } \\
\text { de la asignatura } \\
\text { (R) y dos observa- } \\
\text { dores }(\mathrm{O} 1 \text { y } \mathrm{O} 2) \text {. }\end{array}$ \\
\hline
\end{tabular}

El análisis de la información se realizó a través de una codificación temática (Patton, 2002) manual (sin ayuda de un programa informático) de los datos.

\section{Resultados}

A continuación presentamos los resultados atendiendo a los tres objetivos que se derivan del objetivo general de la investigación y que tienen que ver con: (1) la utilidad de las prácticas en la formación inicial del profesorado, (2) su impacto en el desarrollo profesional de los docentes universitarios y en la mejora de la docencia en la educación superior y (3) propuestas de mejora y su impacto en el currículum y en la organización.

\subsection{Ensayando competencias docentes}

Los resultados de la investigación muestran que las prácticas de las asignaturas han permitido a los estudiantes aprender, ensayar y practicar competencias y habilidades que consideran genuinas de un docente del siglo XXI como es valorar la diversidad en el aula y convertirla en objeto de aprendizaje, dialogar con otros compañeros, llegar a acuerdos, saber escuchar opiniones y puntos de vista muy diferentes a los propios, trabajar en equipo, cumplir plazos de entrega de un trabajo, conocer las 
Aprendiendo el oficio docente. El modelo Lesson Study para mejorar la docencia en la universidad

Adelina Calvo Salvador, Ignacio Haya Salmón y Carlos Rodríguez-Hoyos

potencialidades del aprendizaje entre iguales, etc. De entre todas estas competencias, los estudiantes señalan que el trabajo práctico de aula les ha permitido comprender a fondo el significado de la participación y la posibilidad de generar un clima de aula democrático donde todas las voces y puntos de vista son bienvenidos. Las prácticas les han hecho, también, más conscientes de algunas barreras que existen para la participación en entornos educativos:

Es interesante el hecho de que nos hacían participar a todos (...) porque siempre hay alguno que participa más, que participa al principio y cuando llega tu turno parece que ya se ha dicho todo y piensas "iahora yo que digo?" (...). Es que muchas veces si no te mandan participar, siempre terminan participando las mismas personas en clase, porque otras son más tímidas o más introvertidas y tienen ideas igual que el resto, pero no se atreven a comunicarlas (...) y la dinámica de clase siempre te anima y sabes que vas a tener que hacerlo, sabes que vas a tener que hablar (Grupo de Discusión, GD_Asig2).

En algunos casos, los estudiantes muestran satisfacción por haber podido abordar la tarea del trabajo en equipo como un trabajo verdaderamente colaborativo, más allá del trabajo individual aislado que al final se ensambla en un producto final. En este sentido un estudiante señala:

A mí me ha llamado mucho la atención que en los años que llevamos en la universidad los trabajos decimos que "los hacemos a bulto", es decir, "tú haces esta parte, yo hago esta..." Y en este trabajo es imposible, todos tienen que participar, todos tienen que saber qué se hace, todos. Si falta una persona se nota muchísimo, porque las ideas de uno complementan a las de otro, aunque yo si digo una cosa y a lo mejor otro compañero dice otra, nos podemos ayudar mutuamente, no es como un trabajo teórico convencional, digamos (...). Es un trabajo totalmente distinto a los que venimos haciendo durante la carrera (GD_Asig1).

Experimentar la colaboración como forma de trabajo que posibilita alcanzar objetivos educativos importantes en las prácticas de aula les ha permitido reflexionar sobre la importancia de esta competencia en sus futuros lugares de trabajo, como profesionales de la educación que deben desempeñar una tarea a nivel de aula y de centro: 
[El objetivo de las prácticas era] hacernos conscientes o que sepamos que no sólo tenemos que actuar en el aula, sino que tenemos que actuar a nivel de centro, eso es muy bonito, pero si llegas a un centro y allí no te hace caso nadie, ¿qué haces? (...) Porque se supone que trabajas en colaboración con los demás, ¿no? (...) Si te encuentras tú solo, intentas buscar apoyos. (...) Entonces, yo creo que la finalidad es hacernos conscientes de que tenemos que trabajar en equipo para mejorar un centro (GD_Asig3).

Las prácticas de aula han cumplido el objetivo de lograr que los estudiantes aprendan mejor y comprendan a fondo conceptos clave de las asignaturas. El esfuerzo realizado por los docentes para relacionar los contenidos de orden más teórico con los de orden más práctico parece haber dado sus frutos y permite a los jóvenes aprender algunas herramientas para la acción, comprender algunas sugerencias o ideas sobre cómo abordar los procesos educativos en su futuro docente a partir de buenas prácticas educativas que ya se están llevando a cabo en diferentes centros educativos:

En muchas asignaturas nos faltan esos ejemplos de la vida en el aula y con estas experiencias, siempre puedes pensar lo que harías tú como docente con ellas, puedes incluir diferentes temas y piensas qué podrías trabajar y de qué manera (...). Estas experiencias nos sirven para ponernos en situación de decir, "vale, esto se puede hacer en el aula, se puede hacer de esta manera o tiene estos errores" y como que te pone mucho en esa situación de yo, futura docente, no solamente estoy aprendiendo una teoría que luego no sé cómo voy a trabajar en el futuro (...). A la hora de estudiar has tenido que conectar todo lo que has visto en las experiencias y lo que has visto en clase con la teoría, o sea, todo el rato haciendo esas relaciones, entonces estás aprendiendo conceptos, interiorizándolos mientras realizas los debates en grupo, mientras aprendes a hablar en grupo (GD_Asig2).

La presencia de docentes como observadores en el aula, tal y como es propio del modelo LS, ha permitido que estos tuvieran un rol activo, pues los estudiantes acudían a ellos en busca de tutorización y asesoramiento durante el desarrollo de las prácticas de aula. Esto ha sido valorado muy positivamente por los jóvenes, quienes han podido experimentar los efectos positivos de la metodología de trabajo docente que plantea la existencia de varios docentes en el aula: 
Aprendiendo el oficio docente. El modelo Lesson Study para mejorar la docencia en la universidad

Adelina Calvo Salvador, Ignacio Haya Salmón y Carlos Rodríguez-Hoyos

A mí me ha ayudado muchísimo el hecho de que aparte del profesor de la asignatura, haya habido dos personas más en clase, porque al menos en mi grupo hemos sacado ideas de todos. Cada uno nos ha ayudado en una cosa (GD_Asig1).

Las prácticas de aula y el modelo de LS utilizado para su seguimiento ha permitido al alumnado experimentar que no existe una única forma de organizar los procesos educativos y que el cambio es posible en educación. Al mismo tiempo, se reconoce que el cambio institucional requiere un compromiso personal al sentirse vinculado a las actividades propuestas:

[Lo más importante de las prácticas] ha sido, para mí, sentirme parte implicada en todas esas cosas, no quedarte con la idea de que "bueno, eso se hace así y se debe hacer así"... ¿sabes?, sino que hacernos un poco conscientes de que realmente esas cosas pueden cambiar. Incluso yo le he presentado mi trabajo a mi profesora de prácticas y le pregunté si se podría hacer algo parecido en su centro (GD_Asig3).

Otro de los aspectos destacados por los propios estudiantes es en qué medida las prácticas de aula y el modelo LS aplicado para su mejora, les permite acercarse a cómo pensar una innovación docente, entendiendo que, como profesores en una sociedad cambiante y diversa, ellos están llamados a innovar:

Es como que en educación le damos mucha importancia a la escucha de los niños, a que todo gire en torno a sus intereses y yo ahora me he sentido un poco "niño", porque el que me está educando se está centrando en lo que yo pienso y en lo que yo quiero (...). Yo creo que es muy importante, porque si se toma en cuenta lo que hemos dicho, a lo mejor sirve de ayuda y otras personas podrán dar otras ideas que ayudarán en la siguiente etapa, me parece muy importante y creo que habría que hacerlo en más asignaturas (...). Además es que nos están educando para ser profesoras y tenemos que innovar, es lo que nos dicen en la mayoría de las asignaturas (GD_Asig1).

El proceso de reflexión generado en las aulas ha logrado desbordar los límites de lo propuesto en las asignaturas para alcanzar una reflexión más general sobre cuál debe ser el papel de la universidad en la forma- 
ción de los jóvenes. En este sentido, se señala el rol de la institución de educación superior como generadora de pensamiento crítico y divergente:

Yo pienso que no venimos a la universidad sólo a que nos digan en qué consiste el currículum y cuatro cosas más. Yo creo que llegué a la universidad pensando que me iban a enseñar a pensar, a reflexionar sobre las cosas que nos rodean y más a la hora de trabajar con niños... a darle una vuelta a las cosas, a no prejuzgar lo que ves (...). Estamos aquí para reflexionar, para pensar, para darle bastantes vueltas a las cosas y luego, temas tan complicados como pueden ser el machismo, la igualdad, el maltrato, el poder, etc. poder trabajarlo con los niños (...). La asignatura me ayuda a desarrollarme como persona, como profesora también (GD_Asig1).

\subsection{Creando una comunidad de práctica}

Los docentes implicados han señalado que la experiencia de investigar su práctica les ha hecho más conscientes de algunas cuestiones que, aun sabiendo importantes, no consideraban centrales hasta que las han contrastado con el grupo de estudiantes. Muchas de estas competencias se daban por aprendidas, dado que suele pensarse que se ejercitan en prácticamente todas las asignaturas del plan de estudios desde el primer curso, lo que en ocasiones impide ver los conflictos y los retos que subyacen al ejercicio de esa competencia. Por ejemplo, cuando se plantea trabajar en grupo y más concretamente, cómo elegimos a las personas con las que queremos trabajar:

Quizás hay algo que debo subrayar más para el curso que viene, intentar explicar que el cambio organizacional pasa por un cambio personal y pasa por encontrarse con gente con la que habitualmente no trabajas, porque la realidad del centro es esa, como bien planteaba una alumna en el grupo de discusión. La realidad de un centro es que tú tienes que acordar cosas con personas con las que no te llevas bien en un centro (Entrev_Prof3).

Al mismo tiempo, el encuentro dialógico con los estudiantes que se ha producido en el grupo de discusión ha generado ideas para mejorar la docencia y ha hecho evidentes algunos dilemas de la práctica docente, como la distancia que existe entre lo que se enseña y lo que se aprende: 
Aprendiendo el oficio docente. El modelo Lesson Study para mejorar la docencia en la universidad

Adelina Calvo Salvador, Ignacio Haya Salmón y Carlos Rodríguez-Hoyos

En el grupo de discusión se evidenció la distancia que hay muchas veces entre lo que nosotros como docentes tratamos de hacer y lo que al final ellos narran en ese proceso y construyen sobre lo que ocurre en clase. Esto me hace pensar en cómo establecer puentes entre los significados que nosotros otorgamos a lo que hacemos y lo que ellos interpretan (Entrev_Prof1).

La experiencia de los docentes de planificar conjuntamente y contrastar con otros profesionales sus prácticas de aula, la oportunidad de compartir y discutir entre todos las sesiones observadas, ha generado nuevas visiones sobre sus prácticas, cuestiones a mejorar o reconsiderar en próximos ciclos de investigación y muchas de ellas están orientadas a ampliar la red de trabajo inicial creada en el seno de esta investigación:

[En la práctica de aula] se planteaba la idea de que los propios alumnos piensen qué se puede mejorar en un centro, en función de su experiencia como alumno, de su experiencia en prácticas, de las lecturas hechas en la asignatura, etc. Y eso puede ser un trabajo que comience en segundo curso, que es donde está ubicada esta asignatura, pero quizás esa misma práctica se podría recuperar en tercero, en otra asignatura de la misma área de conocimiento y con la misma orientación. Y al hilo de esto que comentábamos, se me había ocurrido (...) que los estudiantes pudieran explicar al grupo su plan de mejora [práctica de aula] y que vinieran otros profesores a modo de "expertos" y que pudieran dar su opinión sobre el plan (Entrev_Prof3).

Saberse inmerso en el proceso de indagación de la propia práctica y contrastar visiones, saberes, formas de hacer, etc. entre los docentes participantes permite reconocer preocupaciones comunes y diseñar propuestas conjuntas de mejora. El modelo LS se reconoce como una herramienta para mejorar la práctica docente y para reconocer dilemas o situaciones-problema comunes que toman otra dimensión cuando salen del terreno de la preocupación individual del docente:

Parece que van adquiriendo cierta relevancia y tomando forma problemas que identifico como comunes y que a la vez nos permiten, en este entorno más de investigación, más de ensayo, o más seguro, ir pensando. Y yo voy identificando ya cuestiones que tienen que ver con lo que llamamos la distancia entre quien orquesta el proceso de enseñanza-aprendizaje y quien 
participa en él sin orquestarlo, quizá tenemos que empezar a pensar que se tiene que empezar a orquestar de manera más polifónica (Entrev_Prof1).

Los docentes han valorado positivamente el uso del modelo LS para investigar su práctica educativa, no solamente porque esto les ha permitido tener un conocimiento más profundo y complejo sobre cómo se desarrollan las prácticas de aula y su impacto en el aprendizaje de los estudiantes, sino porque al mismo tiempo, los estudiantes han podido experimentar algunos de los grandes principios que, a juicio de estos docentes, deben guiar la enseñanza en todos los niveles educativos como la participación o la mejora educativa:

Yo lo valoro muy positivamente, no solo porque accedes a una explicación de las cosas que están pasando diferente a la tuya y eso siempre es bueno porque te da un punto de vista distinto, te permite objetivar la realidad, sino porque también creo que para ellos, y creo que algunas personas que han estado en el grupo de discusión han hecho esa reflexión, es un ejemplo también de cómo mejorar la participación de los estudiantes en clase... Esto que siempre les decimos a ellos que hay que hacer, que los estudiantes tengan más voz, que se le escuche, intentar enganchar a todo el mundo en la dinámica de clase (...) pues yo creo que ellos ven un ejemplo, (...) les da a ellos algunas ideas o algunas estrategias (Entrev_Prof3).

El desarrollo de las prácticas de aula ha permitido crear un espacio de participación con el alumnado, un lugar donde ejercitar la democracia deliberativa, la construcción conjunta de conocimiento, la posibilidad de intercambiar experiencias y de aprender "con" y "de" los demás. Pese a las evidentes ventajas de esta organización del aula, reconocidas por estudiantes y docentes, se perciben cuestiones dilemáticas de difícil resolución y que evidencian la dimensión ética y política de la educación, como por ejemplo, la evaluación educativa:

Yo creo que uno de los mayores inconvenientes tiene que ver con cómo median los procesos de evaluación que son parte inherente a la labor docente (...). Dicho de otra manera, creo que nunca es posible, en tanto exista la variable evaluación, lograr relaciones mucho más horizontales, porque incluso en la preocupación que tenemos sobre cómo construir espacios de aula más deliberativos, las relaciones de poder son las que son, y creo que eso muchas 
Aprendiendo el oficio docente. El modelo Lesson Study para mejorar la docencia en la universidad

Adelina Calvo Salvador, Ignacio Haya Salmón y Carlos Rodríguez-Hoyos

veces nos veta el acceso a espacios más sombríos, diríamos, en los que ellos en concreto no manifiestan todo lo que querrían y no sólo sobre el docente como profesional, sino sobre la propia institución o sobre cuestiones que tienen que ver con la cultura o los criterios que rigen en la facultad, por ejemplo (Entrev_Prof2).

\subsection{Proponiendo mejoras en el trabajo de aula}

Tanto el desarrollo de las clases teóricas y prácticas, como la participación del alumnado en el proceso reflexivo que propone el modelo LS han permitido que los estudiantes logren un significativo nivel de reflexión sobre el trabajo de aula y el oficio del docente. Se ha ampliado su visión de la educación y se han reconocido algunos condicionantes institucionales para el desarrollo de una práctica docente desde el compromiso de la innovación y la mejora. Es desde este lugar desde donde los jóvenes han señalado algunas mejoras en relación al desarrollo de las clases, aflorando en esos momentos las visiones diferentes sobre la enseñanza y de nuevo, algunos dilemas educativos.

Uno de esos dilemas tiene que ver con conseguir un adecuado equilibrio entre la autonomía de los estudiantes en su trabajo y la directividad de los docentes. En este caso, los estudiantes demandaban pautas más estructuradas para el desarrollo de las prácticas, plazos de entrega pautados (no sólo al final, sino intermedios) o incluso, el acceso a prácticas que otros estudiantes de cursos anteriores hubieran desarrollado en el marco de las asignaturas:

Igual el problema es que estamos acostumbrados a trabajar con pautas más cerradas, entonces claro, en el momento en que te abren todas las pautas... En cuanto no sabes qué poner en un punto ya te ves perdido... [Lo más difícil] fue saber qué tenías que poner en cada punto. Yo eso de que me lo dejen libre, lo llevo muy mal, porque no sé qué tengo que poner y me agobio mucho... las preguntas eran como muy abiertas, muy abstractas, no sabíamos qué contestar, pero que está muy bien que nos hagan pensar. (...) Igual no tenías que dejar a nuestra voluntad que te enseñáramos las prácticas, sino poner unas fechas [de entrega], porque siempre pasa que cuando tienes una fecha límite, es como que cumples... y si no, lo vas dejando y te encuentras la semana de antes estresada (GD_Asig3). 
Por otro lado, los docentes consideraban que el establecimiento de un marco no excesivamente estructurado en el que desarrollar el trabajo de aula podría ayudar a promocionar la autonomía de los estudiantes y trataban de desarrollar varias estrategias para ello:

Yo creo que tener dificultades es parte del proceso de aprendizaje (...). Hay un momento en la asignatura en el que uno se desorienta, porque no sabes cómo abordarlo hasta que das con ello, es lo mismo que cuando tienes que escribir un artículo de investigación o un diario (...). A mí muchas veces me hacían preguntas y yo se las devolvía, porque creo que eso forma parte de tu aprendizaje, tú tienes que tomar decisiones, tienes que decidir por dónde vas a empezar el trabajo, qué concepto te parece más importante (...). Pero es verdad que es tarea del profesor no perder nunca el equilibrio, para que la gente no esté completamente perdida y se desenganche (Entrev_Prof3).

Una segunda cuestión que los estudiantes plantearon como mejora fue la posibilidad de potenciar la ayuda entre iguales, en espacios y tiempos que pudieran estar más pautados por el docente, o en espacios autogestionados por ellos mismos. Así lo reconocía el docente tras la celebración del grupo de discusión:

Voy a intentar organizar un taller voluntario fuera del horario de clase, podrían ser dos, uno vinculado a herramientas de edición de imagen fija y otro vinculado al manejo de herramientas de edición audiovisual, al menos para que las personas que no tienen tantas destrezas puedan trabajar en otro contexto. E intentaré sumar a aquellas personas que en todo el grupo puedan tener más competencias tecnológicas para que dinamicen este tipo de espacios (Entrev_Prof1).

Por último, los estudiantes consideraban que era importante dar visibilidad a los productos educativos generados en sus prácticas, bien a través de una discusión y presentación de los mismos a todos los compañeros de clase (GD_Asig1), bien realizando esta misma presentación a profesionales internos, es decir, profesorado de la facultad de educación o externos, maestros en ejercicio (GD_Asig3 y Entrev_Prof3). Esta discusión pública de sus proyectos cumpliría el doble objetivo de sistematizar y relatar toda la experiencia vivida, así como recoger las aportaciones 
Aprendiendo el oficio docente. El modelo Lesson Study para mejorar la docencia en la universidad

Adelina Calvo Salvador, Ignacio Haya Salmón y Carlos Rodríguez-Hoyos

que otros profesionales podrían hacer de ellos, teniendo como horizonte la mejora del trabajo realizado.

\section{Discusión de resultados y conclusiones}

En este artículo se ha analizado la aplicación del modelo LS para mejorar el aprendizaje de los estudiantes a través del análisis y discusión de las prácticas de aula que han sido tomadas aquí como lecciones de estudio. La metodología utilizada ha permitido, no solamente el trabajo conjunto entre docentes, sino también entre docentes y estudiantes. Se ha promocionado la participación de los jóvenes como "amigos críti$\cos ^{\prime \prime}$, como expertos en su propio aprendizaje, lo que ha favorecido la reflexión sobre su proceso de aprendizaje. Como se ha señalado en otras investigaciones, la participación de los estudiantes es fundamental para mejorar la enseñanza (Ayala y Sánchez, 2018; Cheminais, 2011; Wood y Cakjler, 2016).

En el caso de los estudiantes, las prácticas de aula y el ciclo de reflexión-acción que promueven las LS han generado que durante las clases, los jóvenes hayan podido experimentar, aprender, modificar, poner en juego y reflexionar sobre algunas de las competencias que se consideran fundamentales en el ejercicio de la función docente como la participación en el aula, el trabajo colaborativo como estrategia de mejora educativa, la reflexión sobre cómo aprendemos estableciendo puentes entre los contenidos de orden más teórico y práctico en las asignaturas, las potencialidades de la metodología de dos profesores por aula, así como las posibilidades del cambio y la innovación educativa. Han tomado una mayor conciencia, también, de las posibilidades y límites del trabajo en grupo y de algunos de los principales dilemas que son consustanciales a la profesión de educar. Desde este punto de vista, los estudiantes han alcanzado un aprendizaje profundo. Dado que los aprendizajes realizados dependen de factores contextuales, el enfoque de aprendizaje profundo se asocia con una enseñanza caracterizada por proponer libertar para aprender, apoyar al estudiante, dar un adecuado feedback, así como proponer objetivos claros y relevantes desde el punto de vista de la práctica profesional (Monroy y Hernández Pina, 2014).

Desde la perspectiva de los docentes, se reconocen las potencialidades del modelo LS para promover mejoras en la enseñanza universitaria, 
poniendo en diálogo las visiones de docentes y estudiantes. Se ha buscado la creación de espacios de democracia deliberativa, sin perder de vista cómo están funcionando las relaciones de poder en el aula y los efectos de la evaluación. Al mismo tiempo, reconocen que esta investigación ha permitido hacer partícipes a los estudiantes de un proceso de mejora docente no sólo desde el discurso, sino tomando partido en ella, siendo una pieza fundamental del mismo. La planificación conjunta de las prácticas de aula, su desarrollo, la recogida y el análisis de los datos, así como las propuestas de mejora permiten afirmar que se ha alcanzado un alto grado de colaboración docente y no solamente una mera coordinación con un impacto débil en el trabajo conjunto. Las investigaciones sobre Mejora Escolar demuestran que la colaboración promueve oportunidades para el desarrollo profesional articuladas a partir de la reflexión compartida sobre los problemas y dilemas de la práctica docente. Es así como los docentes pueden aprender conjuntamente a partir de procesos de indagación colectiva basadas en la observación de las clases y en una recogida de datos sistemática (Krichesky y Murillo, 2018).

Docentes y estudiantes han generado, en diálogo, algunas mejoras que se pueden considerar para las futuras ediciones de las prácticas de aula, a la vez que se han evidenciado las dificultades propias de quienes se encuentran a uno y otro lado del proceso de enseñanza-aprendizaje.

En definitiva, los resultados muestran cómo la Investigación-Acción puede ser una estrategia de formación en la educación universitaria, al proponer una forma de entender la profesión en la que el docente investiga su propia práctica con el objetivo de comprenderla, producir conocimiento y transformarla (Cunha, 2015; Martin y Clerc-Georgy, 2015).

\section{Referencias}

Alonso, L. E. (1998). La mirada cualitativa en sociología: una aproximación interpretativa. Madrid: Fundamentos.

Angrosino, M. (2012). Etnografía y observación participante en Investigación Cualitativa. Madrid: Morata.

Ayala, I. y Sánchez, V. (2018). Identidades reflexivas en la universidad: sonidos, imágenes y microrrelatos autobiográficos. Educatio Siglo XXI, 36(1), pp. 215-230.

Barbour, R. (2013). Los grupos de discusión en Investigación Cualitativa. Madrid: Morata. Bolarín, M. J. (2016). La coordinación docente en la universidad: análisis de la situación actual. Educatio Siglo XXI, 34(2), 167-184. 
Aprendiendo el oficio docente. El modelo Lesson Study para mejorar la docencia en la universidad

Adelina Calvo Salvador, Ignacio Haya Salmón y Carlos Rodríguez-Hoyos

Braga, G., Verdeja, M. y Calvo, A. (2018). La Metodología Lesson Study en un Contexto Universitario. Una experiencia para Mejorar las prácticas de Aula. Qualitative Research in Education, 7(1), 87-113.

Caballero, K. y Bolívar, A. (2015). El profesorado universitario como docente: hacia una identidad profesional que integre docencia e investigación. REDU. Revista de docencia universitaria, 13(1), 57-77.

Calvo, A. y Rodríguez-Hoyos, C. (2017). El modelo de las Lesson Study para mejorar la docencia Resultados de una investigación en la universidad de Cantabria (España). En F. J. Murillo (Coord.). Avances en liderazgo y mejora de la educación. Actas del I Congreso Internacional de Liderazgo en Educación, (pp. 264-266). Madrid, RILME.

Cheminais, Rita (2011). Pupil Voice and Action Research. En G. Czerniawski y W. Kidd (Ed.). The Student Voice Handbook: Bridging the Academic/Practitioner Divide, (pp. 341-350). Bradford: Emerald.

Cunha, M. I. (2015). Investigación y docencia: escenarios y senderos epistemológicos para la evaluación de la educación superior. REDU. Revista de docencia universitaria, 13(1), 79-94.

Dudley, P. (2011). Lesson Study development in England: from school networks to national policy. International Journal for Lesson and Learning Studies, 1(1), 85-100.

Dudley, P. (2015). El desarrollo de Lesson Study en Inglaterra en el siglo XXI (2000-2015) y el potencial de una Red Europea. Revista Interuniversitaria de Formación del Profesorado, 84(29.3), 61-79.

Elliot, J. (2015). Las Lesson y Learning Studies y la idea del docente como investigador. Revista Interuniversitaria de Formación del Profesorado, 84(29.3), 29-46.

Escudero, J. M. (2009). El amigo crítico, una posibilidad para la formación en los centros. Compartim. Revista de Formació del Professorat, 4(1-4). Disponible en: http://cefire. edu.gva.es/sfp/revistacompartim/arts4/01_com_amigo_critico.pdf

Flick, U. (2014). El diseño de la Investigación Cualitativa. Madrid: Morata.

Holmqvist, M. (2015). Learning Study. Del estudio de la enseñanza al estudio del aprendizaje. El movimiento de Learning Study en Suecia. Revista Interuniversitaria de Formación del Profesorado, 84(29.3), 47-59.

Hurd, J. y Licciardo-Musso, L. (2005). Lesson Study: Teacher-Led Professional Development in Literacy Instruction. Language Arts, 82(5), 388-395.

Jiménez, J. (2017). La docencia en la educación superior. Cuadernos de Pedagogía, 476, 42-45.

Krichesky, G. y Murillo, F. J. (2018). La colaboración docente como factor de aprendizaje y promotor de mejora. Un estudio de casos. Educación XX1, 21(1), 135-156.

Kvale, S. (2011). Las entrevistas en Investigación Cualitativa. Madrid: Morata.

Lewis, C. (2002). Lesson Study: A Handbook of Teacher-Led Instructional Change. Philadelphia: Research for Better Shools.

Martin, D. y Clerc-Georgy, A. (2015). Use of theoretical concepts in lesson study: an example from teacher training. International Journal for Lesson and Learning Studies, 4(3), 261-273.

Martínez, J. M. (2017). Retomar la renovación de las metodologías educativas. Cuadernos de Pedagogía, 476, 51-55. 
Aprendiendo el oficio docente. El modelo Lesson Study para mejorar la docencia en la universidad

Adelina Calvo Salvador, Ignacio Haya Salmón y Carlos Rodríguez-Hoyos

Martínez Rodríguez, J. B. (2011). La formación docente: una mirada internacional. Cuadernos de Pedagogía, 417, 77-80.

Mas Torelló, O. y Olmos-Rueda, P. (2016). El profesor universitario en el Espacio Europeo de Educación Superior. La autopercepción de sus competencias docentes actuales y orientaciones para su formación pedagógica. Revista Mexicana de Investigación Educativa, 21(69), 437-470.

Monereo, C. y Domínguez, C. (2014). La identidad docente de los profesores universitarios competentes. Educación XX1, 17(2), 83-104.

Monroy, F. y Hernández Pina, F. (2014). Factores que influyen en los enfoques de aprendizaje universitario. Una revisión sistemática. Educación XX1, 17(2), 105-124.

Montero, L. (2018). Relaciones entre teoría y práctica en la formación inicial. Percepciones de formadores y estudiantes de Grado de Maestro en Educación Primaria. Educatio Siglo XXI, 36(2), 303-330.

Patton, M. Q. (2002). Qualitative research and evaluation methods. California, Sage.

Peña, N. y Pérez Gómez, A. (2019). Las disposiciones subjetivas de los docentes en la superación de las resistencias al cambio ante procesos cíclicos de formación basados en la investigación (Lesson Study): Estudio de un caso. Revista Complutense de Educación, 30(2), 569-587.

Pérez Gómez, A. I. y Soto, E. (2011). La mejora de la práctica y la investigación docente. Cuadernos de Pedagogía, 417, 64-67.

Pétriz, F. y Rubiralta, M. (2017). Las misiones de la Universidad. Cuadernos de Pedagogía, 476, 46-50.

Ruè, J. (2015). El desarrollo profesional docente en Educación Superior: agenda, referentes y propuestas para su adopción. REDU. Revista de docencia universitaria, 13 (núm. extraordinario), 217-236.

Soto, E. y Pérez Gómez, A. I. (2015). Lesson Studies: Un viaje de ida y vuelta recreando el aprendizaje comprensivo. Revista Interuniversitaria de Formación del Profesorado, 84(29.3), 15-28.

Soto, E.; Serván, M. J.; Pérez Gómez, A. (2015). Cooperative research as university teacher training. A case study of lesson and learning study. International Journal for Lesson and Learning Studies, 4(1), 56-71.

Soto, E. Serván, M. J. y Caparrós, R. (2016). Learning to teach with Lesson Study: the practicum and the Degree Essay as the scenario for reflective and cooperative creation. International Journal for Lesson and Learning Studies, 5(2), 126 - 139.

Stake, R. (1998). Investigación con estudio de casos. Madrid: Morata.

Wood, P. y Cajkler, W. (2016). A participatory approach to Lesson Study in higher education. International Journal for Lesson and Learning Studies, 5(1), 4-18.

Zabalza, M. A. (2004). Los diarios de clase: documento para estudiar cualitativamente los dilemas prácticos de los profesores. Madrid: Narcea. 
